\title{
A license plate location method combining the license plate location method based on the HSV color space and the license plate location method based on the boundary detection
}

\author{
LIN Wei, DU Hao \\ College of Physics and Information Engineering, Fuzhou University, Fuzhou, China. \\ College of Physics and Information Engineering, Fuzhou University, Fuzhou, China. \\ mqks@fzu.edu.cn,358231384@qq.com
}

Key words: license plate location; HSV color space; boundary detection.

\begin{abstract}
The first step of the license plate automatic recognition is the license plate location,therefore,the license plate location method is very important to the license plate automatic recognition.A license plate location method combining the license plate location method based on the HSV color space and the license plate location method based on the boundary detection is presented in this paper. A total of 216 experimental images are tested in this paper. Through the analysis of experimental results, it's found that this method can achieve the license plate location accurately, and the method's accuracy is 98.6 percent.
\end{abstract}

\section{Introduction}

The license plate location method based on boundary detection[1,3] and the license plate location method based on the color space[4,5] are the most common license plate location methods, according to the license plate color, the second method carries out the subsequent operations , and it performs well, but it will be failed when the plate is dyed with other color. In this paper, the license plate location method based on the HSV color space and the license plate location method based on the boundary detection are combined, and its license plate location accuracy is good.

A license plate location method combining the license plate location method based on the HSV color space and the license plate location method based on the boundary detection

At first,the RGB color space will converted to HSV color space[6,7] ,then, the binarization processing is taken, in the HSV color space, the binarization processing can be done by judging three components values of HSV color space. The value of $\mathrm{H}$ components are different when colors are different, if the value of $\mathrm{S}$ component and the $\mathrm{V}$ component value is fixed, the color will be changed when the value of $\mathrm{H}$ component is changed, Looking over all pixels of the image, value of the pixel will be set to 1 if each component value of the pixel is in the component value range of the color specified, otherwise, the value of pixel will be set to 0 , the Fig.1 is the original image, and the Fig.2 is the binarization processing result of Fig.1.

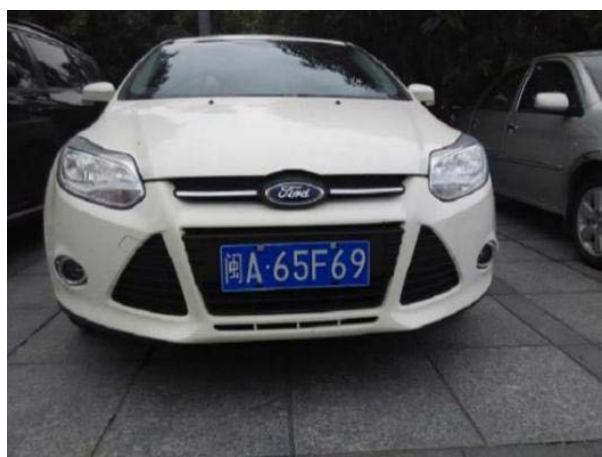

Fig.1

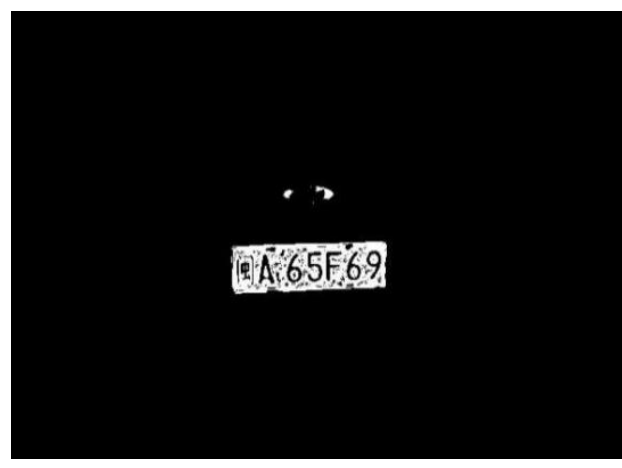

Fig.2 
Then, the morphology operation is taken to obtain license plate candidate areas, the morphology operation used in this method includes morphological closing operation and morphological open operation, after that, peripheral contours of the connected domains can be drawn and minimum tangent rectangles of the drawn connected domains can be calculated, and the minimum tangent rectangle areas are candidate areas of the license plate. After the morphology operation, the peripheral contours can be drawn and displayed on the original image, the result of the morphology operation is shown below in Fig.3, The length and the width of the Chinese license plate are specified, so the ratio of the Chinese license plate length and the Chinese license plate width is almost a constant value, that constant value is assumed to be $\mathrm{M}$, and $\mathrm{x}$ is set as a parameter of allowable error, then the license plate area will be found by verifying the ratio of the minimum tangent rectangle length and the minimum tangent rectangle width. The minimum tangent rectangle area is considered to be the license plate area if the ratio of its length and its width is a value in [(1$\mathrm{x}) \mathrm{M},(1+\mathrm{x}) \mathrm{M}]$, the result is shown below in image Fig.4.

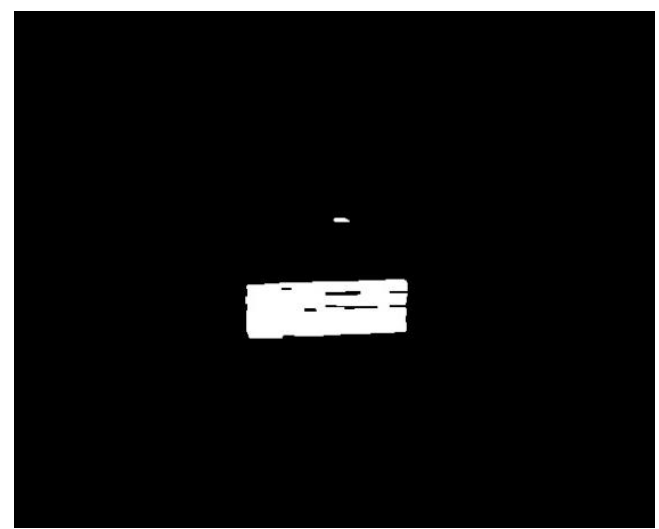

Fig.3

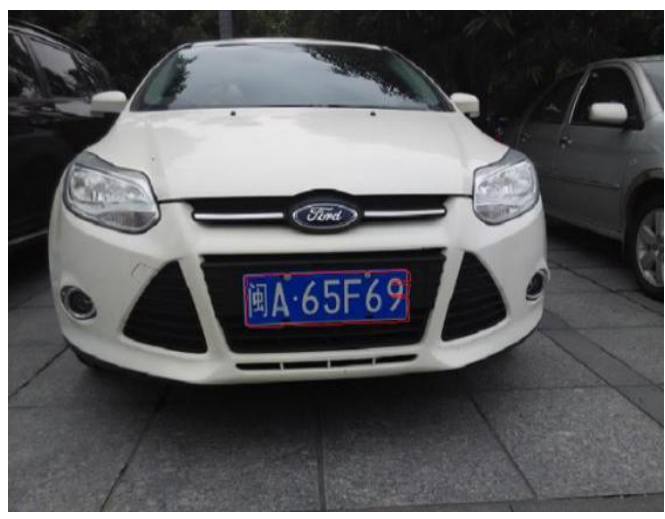

Fig.4

Fig.4 is a successful result of license plate location method base on HSV color space, but the license plate location method base on HSV color space will be failed when every ratio of the minimum tangent rectangle length and the minimum tangent rectangle width isn't a value in [(1$\mathrm{x}) \mathrm{M},(1+\mathrm{x}) \mathrm{M}]$. In the HSV color place, the binarization processing can be done by judging the component value of the HSV color space, the binarization processing result of Fig.5 is shown below in Fig.6,and then, the license plate location method based on the HSV color space is failed.

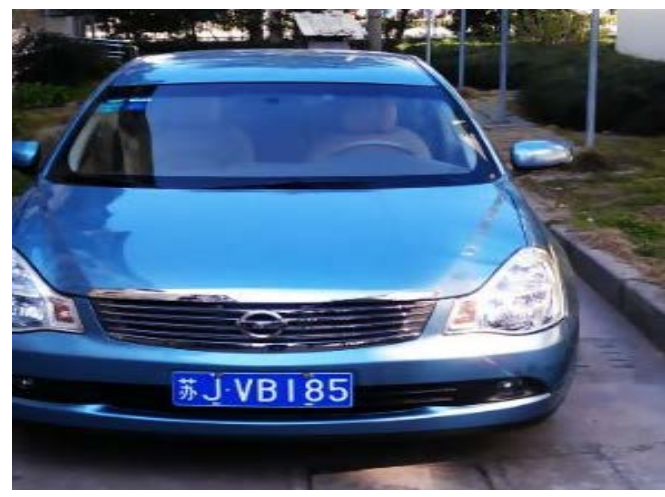

Fig.5

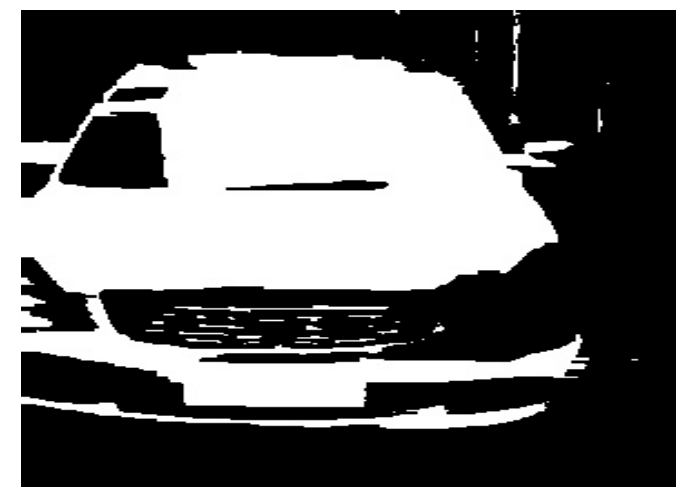

Fig.6

When the license plate location method based on the HSV color space is failed, the license plate location method based on the boundary detection will be followed to finish the license plate location. After some image preprocessings being taken the grayscale image can be acquired. The method will detect the grayscale image edge by using Sobel operator, the result is shown below in the Fig.7, After that, the binarization processing is taken, then, the morphology operation is taken to obtain license plate candidate areas, the result is shown below in image Fig.8, then, the peripheral contours of the connected domains can be drawn and minimum tangent rectangles of the drawn connected domains can be calculated, after the peripheral contours being displayed on the original 
image, the result is shown below in image Fig.9. At last, the license plate location can be achieved by verifing the ratio of the minimum tangent rectangle length and the minimum tangent rectangle width, the result is shown below in image Fig.10.

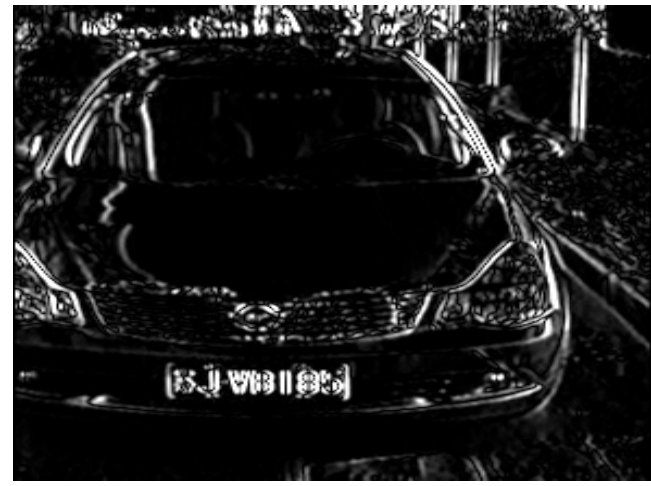

Fig.7

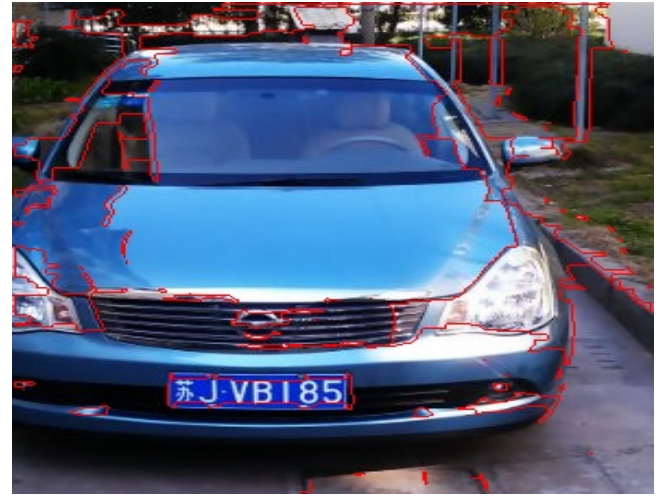

Fig.9

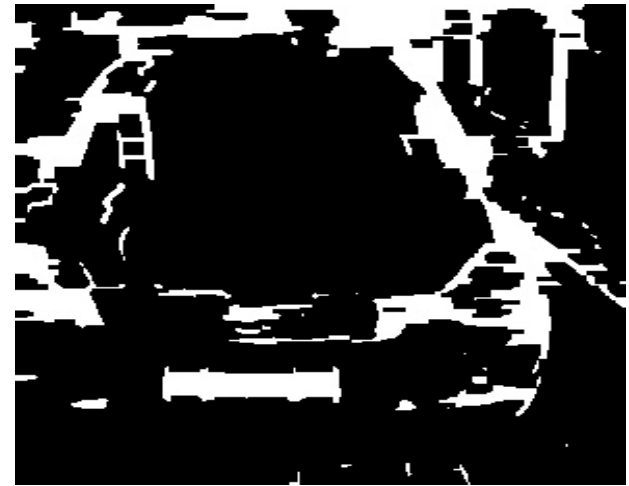

Fig.8

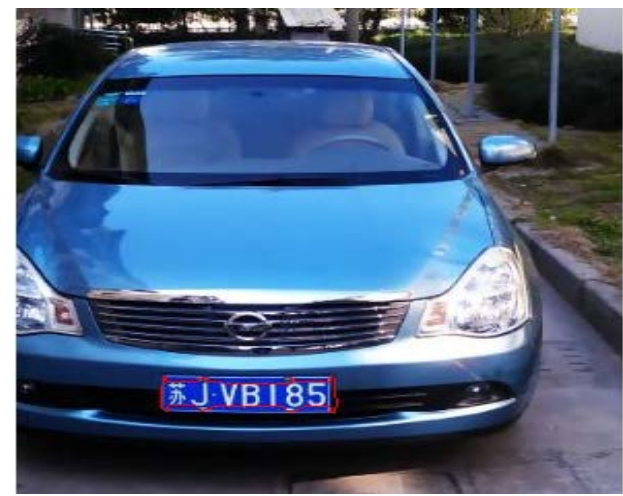

Fig.10

\section{The experiment data and result analysis}

Three license plate location methods are tested in this experiment, and 216 images are used in the experiment, the location accuracy of different license plate location methods are tested out, and the average location times of different license plate methods are tested out too, the experiment result is shown below in the table 1 .

Table 1 the experiment result of three license plate location methods

\begin{tabular}{|l|c|c|c|}
\hline \multicolumn{1}{|c|}{ License plate location method } & $\begin{array}{l}\text { Number of images } \\
\text { being located } \\
\text { successfully }\end{array}$ & $\begin{array}{c}\text { Location } \\
\text { accuracy }\end{array}$ & $\begin{array}{l}\text { Average time of } \\
\text { one image } \\
\text { located [s] }\end{array}$ \\
\hline $\begin{array}{l}\text { The license plate location method base } \\
\text { on the boundary detection }\end{array}$ & 178 & $82.4 \%$ & 0.57 \\
\hline $\begin{array}{l}\text { The license plate location method base } \\
\text { on the HSV color space }\end{array}$ & 205 & $94.9 \%$ & 0.37 \\
\hline $\begin{array}{l}\text { The license plate location method } \\
\text { combining license plate location method } \\
\text { based on the HSV color space and } \\
\text { license plate location method based on } \\
\text { the boundary detection }\end{array}$ & 213 & 0.44 \\
\hline
\end{tabular}

when the license plate location method base on the boundary detection is used, 178 images are located successfully, its location accuracy is $82.4 \%$, and the average time of one image being 
located is $0.57 \mathrm{~s}$. Through the analysis of the experiment result, it is found that this method will be failed when vertical edges appear around the license plate.

When the license plate location method base on the HSV color space is used, the same 216 images are tested. 205 images are located successfully, its location accuracy is $94.9 \%$, and the average time of one image being located is $0.37 \mathrm{~s}$. Through the analysis of the experiment result, it is found that this method will be failed when the license plate color is same with the car color.

When the license plate location method combining the license plate location method based on the HSV color space and the license plate location method based on the boundary detection is used. 213 images are located successfully, its location accuracy is $98.6 \%$, and the average time of one image being located is $0.44 \mathrm{~s}$. Through the analysis of the experiment result, this method can solve many problems that the license plate location method based on color space can't solve, and its license plate location accuracy is also improved a lot.

\section{Summary}

A license plate location method is presented in this paper, it combines the license plate location method based on the HSV color space and the license plate location method based on the boundary detection. This license plate location method can solve many problems that the license plate location method based on the color space and the license plate location method based on the boundary detection can't solve. It can make full use of the license plate color information and achieve the license plate location accurately and efficiently, and the license plate location effect of this method is good.

\section{Acknowledgements}

We acknowledge the financial support for this work from the Key Project Science and Technology Cooperation of Fujian Province [No. 2013I0003] and the School Fund of Fuzhou University [No. 022446].

\section{References}

[1] Zhuo Junfei, Hu Yu. The research on license plate location algorithm based on boundary detection and projection method [J]. Bulletion of Science and Technology,2010,26(3):438-441

[2] Dai Hongxia, Fang Zhe, Zhao Li. The study of image search based on shape feature[J]. Electron Devices,2010,33(6):762-765.

[3] Shi Wen, DU Yuren. The license plate location method based on mathematical morphology[J]. Journal of YangZhou Universith(Natural Science Edition),2010,13(3):69-73.

[4] Zheng Chengyong. A new license plate location method in RGB color space[J]. Journal of Image and Graphics A,2010,15(11):1623-1628.

[5] Nie HongYin, Zhou Weidong, Liu Hui. A new license plate location in color models and comprehensive feature[J]. Computer Engineering and Applications.2010,46(12):221-223.

[6] Zhu Yongzhen, Men Qinghu, Pu Jie, The traffic light automatic recognition based on HSV color space and shape feature[J]. Video Engineering,2015,39(5):150-154.

[7] Yuan Fenjie, Zhou Xiao, Ding Jun. The realization of conversion algorithm between RGB color space and HSV color space based on FPGA[J]. Electron Devices,2010,33(4):493-497. 